\title{
Análise do potencial pozolânico da cinza de casca de arroz (CCA) através da técnica de Refinamento de Rietveld
}

\author{
Analysis of potential pozzolanic ash from \\ rice husk (CCA) using the technique \\ of Rietveld refinement.
}

Luciana de Nazaré Pinheiro Cordeiro ${ }^{1}$, Ângela Borges Masuero ${ }^{2}$, Denise Carpena Coitinho Dal Molin ${ }^{3}$

\footnotetext{
${ }^{1}$ Laboratório de Engenharia Civil - FEC/ITEC/UFPA - CEP: 66075-110, Belém, PA e-mail: lucianapc@ufpa.br

${ }^{2,3}$ Núcleo Orientado para a Inovação da Edificação - DEC/PPGEC/UFRGS - CEP: 90035-190, Porto Alegre, RS e-mail: angelamasuero@ufrgs.br; dmolin@ufrgs.br
}

\section{RESUMO}

É um consenso que a cinza de casca de arroz é um resíduo agrícola com comprovada eficiência pozolânica. Contudo, por se tratar de um resíduo, apresenta problemas de variabilidade decorrente dentre outras coisas de sua origem e dos diferentes processos de geração, fator este que dificulta sua comercialização. Hoje em dia, a determinação da atividade pozolânica do resíduo e determinada pela NBR 5752 ${ }^{[3]}$ (ABNT, 2012) e NBR $5751^{[2]}$ (ABNT, 2012). A desvantagem com relação a estes ensaios é o tempo que se leva para ter uma resposta. Nesse sentido, este trabalho visa analisar a eficiência do refinamento de Rietveld para avaliar a pozolanicidade através do parâmetro denominado de índice de amorfismo. Por meio desse refinamento é possível dizer, de forma quantitativa, a porcentagem de elementos reativos que o resíduo apresenta, operadores com conhecimento da técnica de análise levam em torno de dois dias para apresentar os resultados. Para avaliação dessa técnica, as cinzas foram produzidas através da queima em diferentes temperaturas $(500,600,800,900$ e $1100{ }^{\circ} \mathrm{C}$ ). Fixou-se o tempo de queima do material em três horas e a taxa de aquecimento em $5{ }^{\circ} \mathrm{C} / \mathrm{min}$, e resfriamento lento. Posteriormente realizou-se a moagem da cinza e quantificação do índice de amorfismo por meio de refinamento Rietveld. Os resultados demonstraram que o índice de amorfismo pode ser utilizado como parâmetro de controle de cinzas residuais, visto que cinzas com elevado índice de amorfismo apresentam uma boa pozolanicidade.

Palavras-chave: cinza de casca de arroz, índice de amorfismo, pozolana, refinamento Rietveld.

\begin{abstract}
Rice husk ash (RHA) is an agricultural by-product with well-known pozzolanic properties. However, the widespread commercial use of RHA is hindered by the lack of consistency between batches of this material and by the lack of quick and inexpensive quality control parameters. This study presents some basic principles for the quantification of the amorphous phases of RHA with the assumption that mineralogic characterization with X-ray diffraction can be a good quality control method for use residual or controlled ashes. The samples in this study were prepared with a lithium fluoride $(\mathrm{LiF})$ standard and phase quantification was carried out using Rietveld's refinement method. The analysis of the phases was successfully conducted and results were satisfactory, with maximum relative standard deviation of $5 \%$. These results show that quantitative analysis by Rietveld refinement can be used in the characterization of construction waste.
\end{abstract}

Keywords: rice husk ash, Rietveld's refinement, amorphous materials, construction waste.

\section{INTRODUÇÃO}

A cinza de casca de arroz é um material considerado há muito tempo como uma superpozolana, suas características estão associadas a diferentes fatores, dentre eles destacam-se a composição química da casca e a con- 
dição de queima adotada.

Sensale [17] (2006), foi uma das autoras que observou que as cinzas são predominantemente de natureza silicosa; e que os teores de sílica estão entre 87 e $95 \%$. No relativo à composição mineralógica, Hara et al [12] (1989), analisando as CCA provenientes de diferentes temperaturas de queimas, observou as três principais formas cristalinas da sílica que são quartzo, cristobalita e tridimita. Essas formas cristalinas da sílica possuem estruturas muito diferentes, tendo cada uma um campo de estabilidade bem definido sob condições de equilíbrio.

Como a reatividade da sílica está relacionada entre outras coisas com o polimorfismo dos silicatos, o conhecimento básico destas transformações torna-se interessante, uma vez que contribui para o direcionamento na escolha de temperaturas quando se desejam produzir CCA com interesse comercial.

A difratometria de raios X (DRX) foi a técnica utilizada na caracterização dos materiais, uma vez que permite a identificação dos polimorfos da sílica, bem como a identificação de outros minerais. O método de Rietveld foi a ferramenta usada para analisar as estruturas dos materiais cristalinos, a partir da DRX, uma vez que permite a quantificação de suas fases. Em termos de aplicabilidade o método pode ser usado na análise quantitativa de fases, nos ajustes de parâmetros de células e nos estudos estruturais tais como: determinação de tamanho de cristalitos, distribuição de cátions, incorporação de átomos e formação de vacâncias, posições atômicas e posições de ocupação.

A grande vantagem do método de Rietveld é que utiliza todos os pontos do espectro da difração de raios $\mathrm{X}$, além disso, essa técnica permite a determinação da quantidade de material amorfo. Para isto, basta adicionar uma quantidade conhecida de padrão interno, em geral 10 a $15 \%$ em massa. O padrão interno deve ser perfeitamente cristalino, e não estar entre as fases identificadas na amostra original, e de preferência ser de fácil refinamento. A diferença entre a soma das frações em peso dos componentes cristalinos e a unidade, dá a fração em peso total dos componentes amorfos. Os dados quantitativos são deduzidos a partir de fatores de escalas, após a superposição e minimização das diferenças de espectros medidos e calculados a partir da modelagem de cada uma das fases da mistura (NEUMANN [14], 2004).

Com relação aos indicadores numéricos que servirão de parâmetro de qualidade, tem-se o índice ponderado (Rwp) que deve ser analisado quando se deseja verificar se o refinamento está convergindo. Se este valor estiver diminuindo ao longo dos ciclos de refinamento, significa que o mesmo está sendo bem sucedido. Outro indicador adotado é o índice de Bragg (Rp) que mede a qualidade do ajuste entre as intensidades integradas observadas e calculadas. E o índice esperado (Rexp) que mede a qualidade das intensidades coletadas. Valores de Rwp e Rexp próximos, significa que os mesmos apresentam uma mesma tendência, sendo assim é possível considerar como satisfatório o refinamento. Por fim, será considerado satisfatório o refinamento cujo o goodness-of-it (GOF), valor equivalente da relação entre o Rwp e Rexp, for menor que 1,7. Na prática, valores inferiores a 5 refletem um refinamento otimizado (SALVADOR[16], 2005; PAULUK[15], 2008).

O objetivo deste trabalho é apresentar a quantificação das fases amorfas de CCA, produzidas em diferentes temperaturas, utilizando para tal a metodologia proposta por Rietveld. A pesquisa pretende demonstrar que a caracterização mineralógica por DRX, em conjunto com análises quantitativas de minerais amorfos, tais como o método de Rietveld, podem ser usada como parâmetro para a avaliação do potencial pozolânico de cinzas residuais.

\section{MATERIAIS E MÉTODOS}

\subsection{Materiais}

O cimento utilizado nesta pesquisa foi o cimento Portland CPN 40, de procedência uruguaia, similar ao CPI da indústria brasileira e equivale ao cimento de classe IIA (ASTM ${ }^{[5]} \mathrm{C} 150 / \mathrm{C} 150 \mathrm{M}, 2012$ ), a massa específica desse material é de $3,08 \mathrm{~g} / \mathrm{cm}^{3}$. O mesmo foi escolhido por ser o cimento mais puro disponível no mercado, livre de pozolanas e/ou adições.

O agregado miúdo utilizado tem dimensões padronizadas em quatro faixas granulométricas - \#1,2 $\mathrm{mm}$; \#0,6 mm; \#0,3 mm e \#0,15 mm, representando cada uma 25\% do agregado miúdo.

A origem da areia é quartzosa oriunda do rio Jacuí, disponível comercialmente na região de Porto Alegre/RS/Brasil. Em termos de caracterização física a mesma apresenta diâmetro máximo e massa específica iguais respectivamente a $2,4 \mathrm{~mm}$ e $2,6 \mathrm{~kg} / \mathrm{dm}^{3}$, respectivamente.

A água utilizada para a mistura das argamassas foi proveniente do Departamento Municipal de Águas e Esgoto (DMAE) de Porto Alegre. 
As cascas de arroz utilizadas nessa pesquisa provém de um engenho localizado no município de Viamão/RS, e correspondem a um resíduo proveniente de uma mesma safra. A produção das cinzas foi feita em laboratório, a fim de se obter um controle maior sobre o material produzido.

Com base em pesquisa bibliográfica (BARTHA E HUPPERTZ [7], 1977; DASS [10], 1984; CORDEIRO [8], 2006) optou-se em trabalhar com as seguintes temperaturas de queima $500{ }^{\circ} \mathrm{C}, 650{ }^{\circ} \mathrm{C}, 800{ }^{\circ} \mathrm{C}$, $950{ }^{\circ} \mathrm{C}$ e $1100^{\circ} \mathrm{C}$. Buscava-se dessa forma produzir cinzas com diferentes graus de desarranjo cristalino. $\mathrm{O}$ ciclo de queima empregado foi o lento, com uma taxa de aquecimento de $5^{\circ} \mathrm{C} / \mathrm{min}$ e um patamar de queima de três horas, ou seja, ao atingir a temperatura desejada o mesmo permanecia nesta temperatura por três horas. $\mathrm{O}$ resfriamento adotado foi o lento, sendo realizado no próprio forno.

A queima foi feita na forma de pilhas, colocando-se cerca de $700 \mathrm{~g}$ de cascas por queima, tomando o cuidado de formar pilhas com alturas de $7 \mathrm{~cm}$. No final de cada queima tinha-se cerca de $140 \mathrm{~g}$ de cinzas, ou seja, do total queimado, produzia cerca de $20 \%$ de cinzas.

Após a queima, realizava-se o processo de cominuição das cinzas através da moagem desses materiais em moinhos de bolas horizontal com jarros de porcelanas de diâmetro de $35 \mathrm{~cm}$ e rotação de $40 \mathrm{rpm}$. O parâmetro de controle da moagem foi o diâmetro médio das cinzas, que tinham que ser menor ou igual a $10 \square \mathrm{m}$. A razão entre carga dos corpos moedores e quantidade de cinza foi mantida constante ao longo de todo o processo a uma proporção de 1:5 (cinzas:bolas). A escolha do proporcionamento foi baseada nos resultados obtidos por Pouey, 2006. Os diâmetros das bolas moedoras variavam entre 10 e $20 \mathrm{~mm}$. Sendo que a quantidade de cinza moída por vez, foi de 300 gramas de cinza. Antes da caracterização e análise, as cinzas foram misturadas a fim de garantir a homogeneização. A caracterização química foi feita por fluorescência de raios $\mathrm{X}$, o ensaio baseia-se no princípio de que a absorção de raios $\mathrm{X}$ por parte do material provoca excitação dos átomos que permite a radiação secundária chamada fluorescência. Os raios emitidos têm um comprimento de onda característico para cada um dos elementos que constituem a amostra, na proporção dos elementos presentes. A caracterização mineralógica foi feita por difração de raios X. A partir dos resultados da difração efetuou-se a análise qualitativa e quantitativa dos minerais.

\subsection{Métodos}

O índice de amorfismo é o parâmetro que mede a quantidade de elemento reativo, presente no material, mostrando-se de extrema importância para argamassas compostas por cimento e pozolana.

Na presente pesquisa foi avaliada a quantidade de sílica reativa contida na cinza de casca de arroz calcinada em diferentes temperaturas. Pretendia-se com essa quantificação, analisar até que ponto a variação de temperatura influenciava quantitativamente nas características mineralógicas do material.

Para quantificação de materiais amorfos adicionou-se um mineral conhecido, perfeitamente cristalino, denominado de padrão interno. A adição deste material visa facilitar o refinamento de materiais predominademente amorfos. Nessa pesquisa, optou-se pela fluorita de litio (LiF), que foi misturada a CCA. A quantidade de $\mathrm{LiF}$ foi de $10 \%$ sobre a massa da CCA.

Os difratogramas foram coletados em modo passo a passo $(0,02$ graus $/ \mathrm{s})$, em intervalos $2 \theta$ de $2^{\circ}$ a $72^{\circ}$. Foi utilizado o difratômetro de raios X da marca Philips MPD 1880-PW1710, pertencente ao LCT-EPUSP. As fendas de divergência, recepção e espalhamento correspondem aos seguintes valores, respectivamente, $1 / 2$ o $, 0,2 \mathrm{~mm}, 1^{1 / 2}{ }^{\circ}$. Os dados foram analisados através do programa X Pert High Score. A análise das fases cristalinas presentes na amostra foram comparadas com os difratogramas disponíveis nos bancos de dados de referência do ICSD (Inorganic Crystal Sctruture Database) e ICSD (International Center for Difraction Data). Nos quais encontram-se fichas contendo informações que auxiliam na identificação das fases. Uma fez realizada a análise qualitativa, seleciona-se no banco de dados as fichas correspondentes às fases identificadas e realiza-se a análise quantitativa.

Os parâmetros de refinamento selecionados incluem os fatores de escala, o deslocamento da amostra, os parâmetros de rede de cada fase, os parâmetros que definem a largura e o perfil dos picos calculados e a função de orientação preferencial e assimetria.

Neste trabalho adotou-se o método do padrão interno para a determinação de fase amorfa em compostos parcialmente cristalinos. $\mathrm{O}$ padrão interno utilizado $(\mathrm{LiF})$ foi mantido numa proporção fixa conhecida, o que possibilitou a determinação da fase amorfa através do cálculo de cristalinidade, na qual a soma das áreas de picos de compostos cristalinos e a área líquida do halo amorfo são medidos. A ordem de refinamento adotada nesta pesquisa consistia em iniciar pelo fator de escala e pela condição de deslocamento da amostra. A segunda etapa consistia no refinamento dos parâmetros de rede, seguido dos parâmetros da largura à meia altura. Um vez ajustados esses parâmetros dava-se continuidade ao processo refinando a orientação preferen- 
cial, e as posições atômica. O último parâmetro a ser refinado eram os parâmetros referentes a assimetria. Tomando-se o cuidado de que para um mesmo difratograma o refinamento fosse repetido.

\section{ANÁLISE E DISCUSSÃO DOS RESULTADOS}

\subsection{Caracterização}

A tabela 1 apresenta os resultados da composição química da CCA produzida. Percebe-se que todas as cinzas produzidas apresentam uma quantidade de $\mathrm{SiO} 2$ superior a $80 \%$. Tem-se também que os teores de álcalis e o teor de perda ao fogo não ultrapassam os máximos estabelecidos pela NBR 12653 ${ }^{[1]}$ (ABNT, 2012) e ASTM C618 ${ }^{[6]}$ (2012), parâmetro satisfatório, uma vez que esses constituintes em grandes quantidades podem vir a prejudicar o desempenho dos produtos à base de cimento do ponto de vista durabilidade (NEVILLE [13], 1997).

Tabela 1: Composição química das CCA produzidas em diferentes temperaturas

\begin{tabular}{|c|c|c|c|c|c|c|c|c|c|}
\hline $\begin{array}{l}\text { Temperatura de } \\
\text { queima } \\
\text { CCA }\end{array}$ & $\mathrm{SiO}_{2}$ & $\mathbf{A l}_{2} \mathbf{O}_{3}$ & $\mathrm{Fe}_{2} \mathrm{O}_{3}$ & $\mathrm{CaO}$ & MgO & $\mathrm{SO}_{3}$ & $\mathrm{Na}_{2} \mathrm{O}$ & $\mathbf{K}_{2} \mathbf{O}$ & PF \\
\hline $500^{\circ} \mathrm{C}$ & 89,58 & \multirow{5}{*}{ ND } & 0,17 & 1,34 & 1,02 & \multirow{5}{*}{$\mathrm{ND}^{*}$} & \multirow{5}{*}{ ND* } & 2,78 & 4,29 \\
\hline $650^{\circ} \mathrm{C}$ & 85,08 & & 0,38 & 1,23 & 0,96 & & & 2,59 & 4,31 \\
\hline $800^{\circ} \mathrm{C}$ & 90,94 & & 0,18 & 1,24 & 1,06 & & & 2,24 & 2,43 \\
\hline $950^{\circ} \mathrm{C}$ & 90,86 & & 0,16 & 1,28 & 1,04 & & & 2,23 & 2,38 \\
\hline $1100^{\circ} \mathrm{C}$ & 91,94 & & 0,21 & 1,40 & 1,14 & & & 2,15 & 0,43 \\
\hline
\end{tabular}

*ND: não determinado

No âmbito de caracterização mineralógica por DRX, as composições mineralógicas das amostras analisadas encontram-se na tabela 2 , o sombreamento representa os minerais presentes em cada amostra.

Tabela 2: Composição mineralógica das CCA produzidas em diferentes temperaturas

\begin{tabular}{c|c|c|c|c|c|c|c}
\hline FASES & Fórmula Química & ICDD* & CCA $_{\mathbf{5 0 0}}$ & CCA $_{\mathbf{6 5 0}}$ & CCA $_{\mathbf{8 0 0}}$ & CCA $_{\mathbf{9 5 0}}$ & CCA $_{\mathbf{1 0 0}}$ \\
\hline Quartzo & $\mathrm{SiO}_{2}$ & $33-1161$ & & & & & \\
\hline Calcita & $\mathrm{CaCO}_{3}$ & $05-0586$ & & & & & \\
\hline Plagioclásio & $\mathrm{NaAlSi}_{3} \mathrm{O}_{8}$ & $20-0554$ & & & & & \\
\hline Cristobalita & $\mathrm{SiO}_{2}$ & $82-0512$ & & & & & \\
\hline Cristobalita $\alpha$ & $\mathrm{SiO}_{2}$ & $76-0941$ & & & & & \\
\hline Feldspato Alcalino & $\mathrm{KalSi}_{3} \mathrm{O}_{8}$ & $10-0479$ & & & & & \\
\hline
\end{tabular}

*ICDD (International Center for Diffraction Data): ficha padrão de identificação dos minerais

Observa-se que todas as pozolanas contém materiais cristalinos. Traços de quartzo são vistos em todas as amostras. Picos de cristobalitas tornam-se visíveis a partir da CCA800. Na CCA1100, verifica-se que a cristobalita sofreu uma transformação, modificando para sua forma de cristobalita $\alpha$. Nas figuras de 1 a 5 têm -se os difratogramas obtidos, e os respectivos minerais identificados.

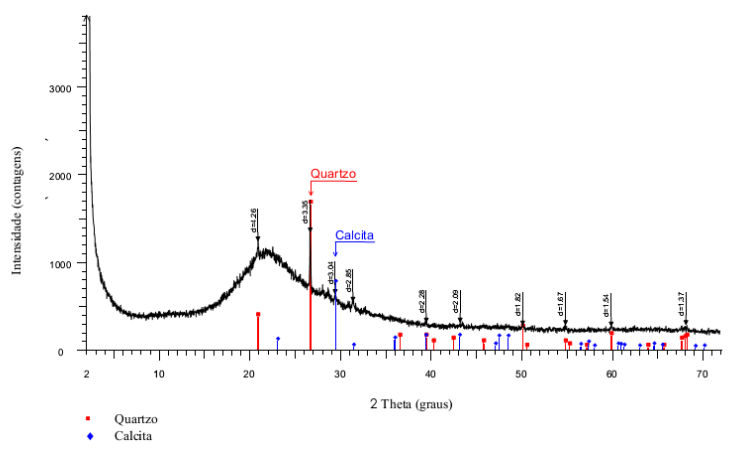

Figura 1: Difratograma da CCA gerada pela queima da casca de arroz a $500^{\circ} \mathrm{C}$

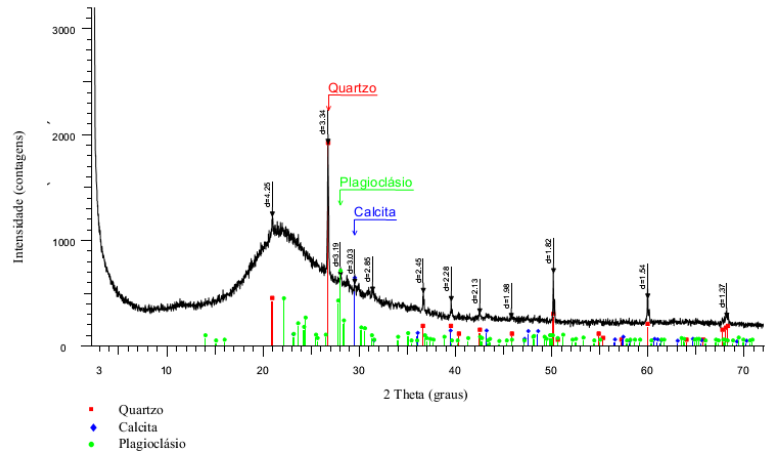

Figura 2: Difratograma da CCA gerada pela queima da casca de arroz a $650^{\circ} \mathrm{C}$ 


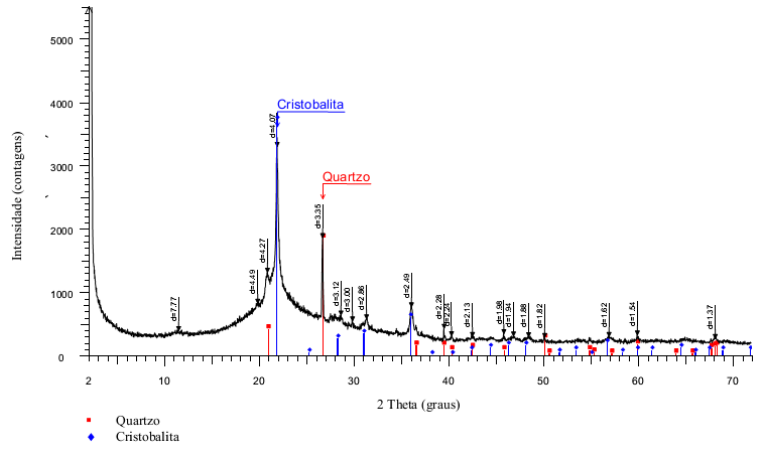

Figura 3: Difratograma da CCA gerada pela queima da casca de arroz a $800{ }^{\circ} \mathrm{C}$

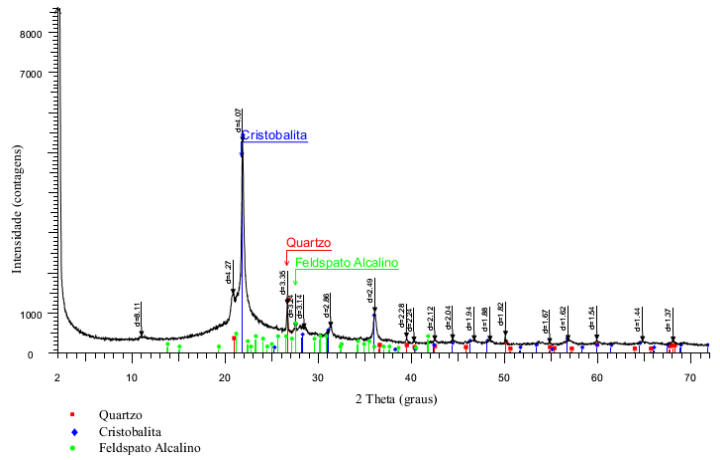

Figura 4: Difratograma da CCA gerada pela queima da casca de arroz a $950{ }^{\circ} \mathrm{C}$

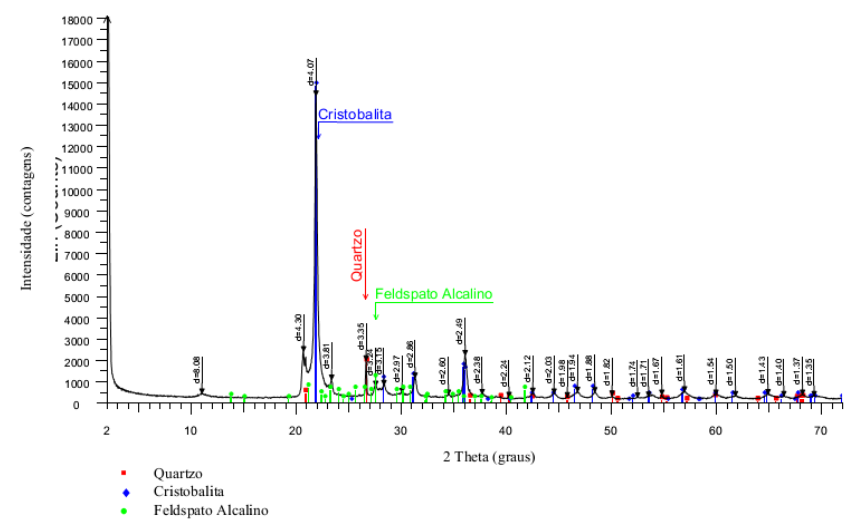

Figura 5: Difratograma da CCA gerada pela queima da casca de arroz a $1100{ }^{\circ} \mathrm{C}$

No que diz respeito ao indicativo de compostos amorfo, verifica-se no espectro da CCA500 um pico largo entre $15^{\circ}$ e $36^{\circ}(2 \theta)$. Este alargamento vai diminuindo a medida que a temperatura aumenta, demonstrando o rearranjo dos compostos cristalinos. No espectro da CCA800 é nítida a verificação da transformação das fases, observando-se a redução do halo amorfo e a formação do pico de cristobalita, que vai se tornando mais definido à medida que a temperatura vai aumentando. Até então, uma análise qualitativa do DRX da figura 4 levaria a crer que o material em questão não apresenta um potencial reativo em função de sua cristalinidade.

Com relação à detecção de alguns minerais, tais como feldspato alcalino, calcita e plagioclásio, podese dizer que a avaliação é bastante complexa; tendo em vista a baixa intensidade de seus picos, que se encontram distribuídos bem próximo do ruído de fundo dos espectros difratométricos.

\subsection{Refinamento Rietveld}

Os resultados do refinamento dos dados obtidos a partir da análise de DRX da amostra de CCA produzidas em diferentes temperaturas são apresentados graficamente nas figuras 6 a 9 .

As linhas em azul representam o difratograma calculado empregando-se o método de Rietveld; e as linhas em vermelho representam o difratograma obtido pela DRX. Na base do gráfico tem-se uma reta em vermelho que representa o desvio entre o calculado e o real. 

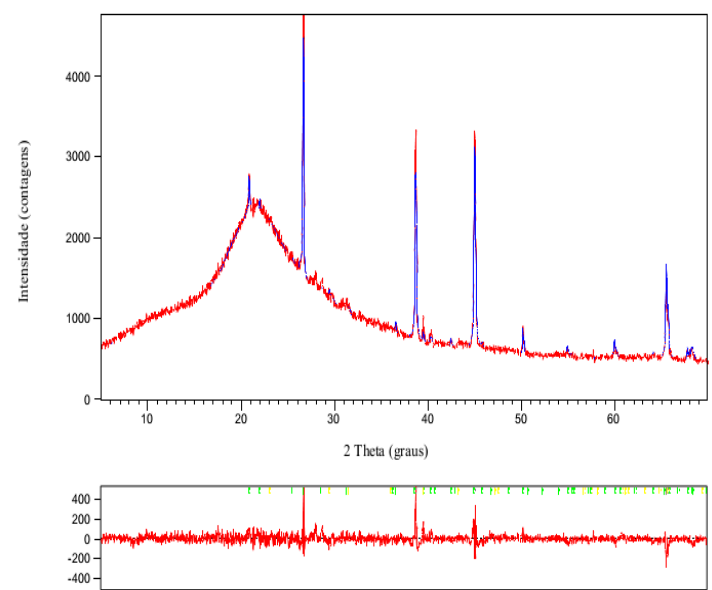

Figura 6: Refinamento pelo método de Rietveld da CCA $500^{\circ} \mathrm{C}$ e ruído obtido

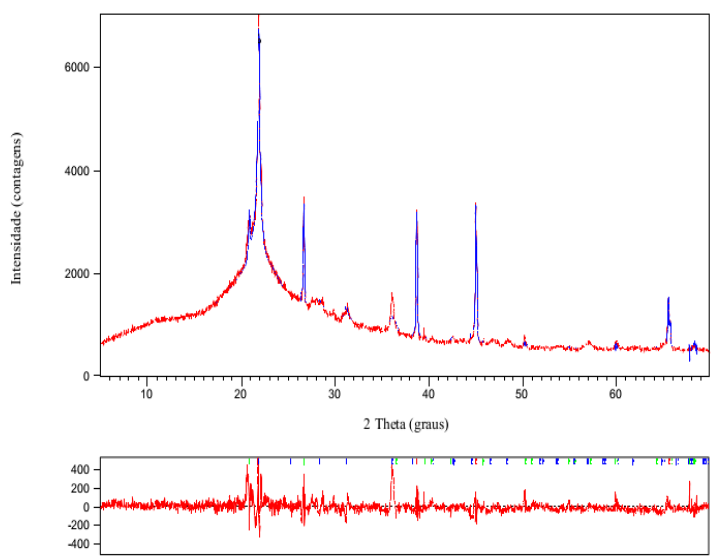

Figura 8: Refinamento pelo método de Rietveld da CCA $800^{\circ} \mathrm{C}$ e ruído obtido

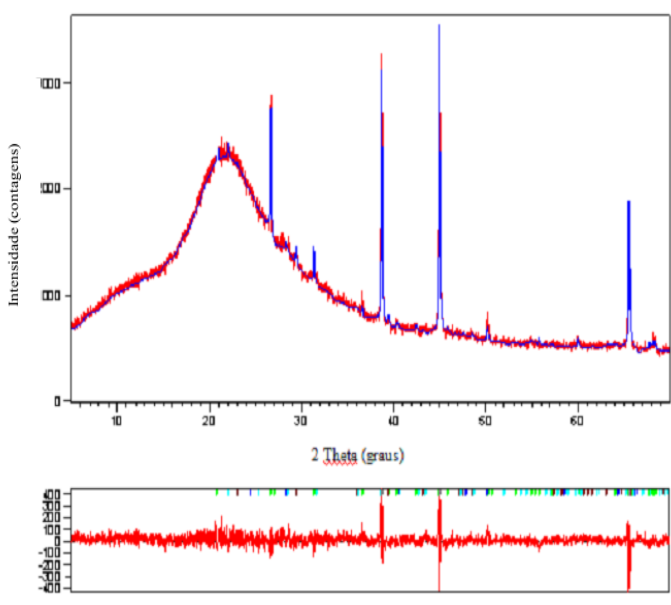

Figura 7: Refinamento pelo método de Rietveld da CCA $650^{\circ} \mathrm{C}$ e ruído obtido
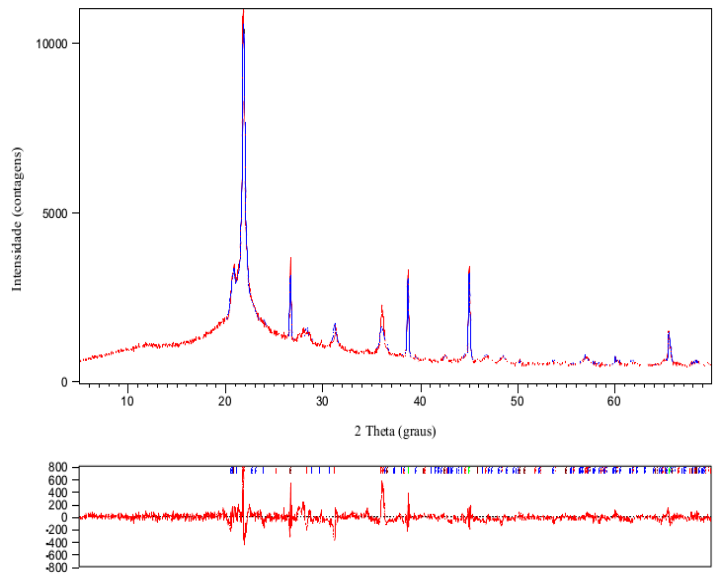

Figura 9: Refinamento pelo método de Rietveld da CCA $950^{\circ} \mathrm{C}$ e ruído obtido

De forma geral os desvios observados graficamente nas figuras 6 a 9 apresentam em sua maioria uma linha contínua, com alguns ruídos; mas que em termos visuais define o ajuste como satisfatório. Rietveld.

A tabela 3 apresenta os valores dos parâmetros de perfil instrumental obtidos pelo refinamento de

Tabela 3: Condições instrumentais adotadas para refinamento de Rietveld

\begin{tabular}{c|c|c|c|c}
\hline \multirow{2}{*}{$\begin{array}{c}\text { Temperatura de queima } \\
\text { CCA }\end{array}$} & \multicolumn{4}{|c}{ Indicadores Numéricos (\%) } \\
\cline { 2 - 5 } & GOF & $\mathbf{R p}$ & $\mathbf{R w p}$ & $\mathbf{R e x p}$ \\
\hline $500^{\circ} \mathrm{C}$ & 1,66 & 3,18 & 4,33 & 3,36 \\
\hline $6500^{\circ} \mathrm{C}$ & 1,95 & 3,23 & 4,65 & 3,32 \\
\hline $800^{\circ} \mathrm{C}$ & 3,19 & 4,59 & 6,35 & 3,19 \\
\hline $9500^{\circ} \mathrm{C}$ & 3,69 & 4,41 & 5,99 & 3,11 \\
\hline
\end{tabular}

*GOF (goodness of it): índice equivalente ao qui-quadrado estatístico; Rp: índice de Bragg; Rwp: índice ponderado; Rexp: índice esperado

Com relação ao GOF observa-se que quanto maior o número de fases maior a complexidade do refinamento. As CCA500 e CCA650 apresentaram valores mais próximos ao de um refinamento perfeito que as demais pois apresentavam um número menor de fases. Para alcançar o mesmo comportamento nas demais amostras, seria necessário inserir no arquivo o modelo de todas as fases existentes, aumentando-se com isso a complexidade do refinamento, para se obter um menor ajuste. Contudo, como o desejado era um refinamento 
otimizado aceitou-se os resultados obtidos.

Com relação ao Rp e o Rexp, parâmetros que medem a qualidade do ajuste entre as intensidades, pode-se dizer que ambos apresentam a mesma tendência, demonstrando que o refinamento é de boa qualidade. No que diz respeito aos resultados obtidos pelo Rwp percebe-se que os mesmos encontram-se com valores consideravelmente aceitáveis; variando de 4,33 a $6,35 \%$, bem inferior ao valor máximo permitido que é de $15 \%$.

Com relação à quantidade de material amorfo, pode-se dizer que as cinzas de cascas de arroz, para estas condições de produção, apresentaram uma grande quantidade de material amorfo, conforme apresentado na tabela 4.

Tabela 4: Teor de amorfismo para CCA calcinadas em diferentes temperaturas

\begin{tabular}{c|c|c|c|c}
\hline & $\mathbf{C C A}_{500}$ & $\mathbf{C C A}_{\text {650 }}$ & $\mathbf{C C A}_{\mathbf{8 0 0}}$ & CCA $_{\mathbf{9 5 0}}$ \\
\hline Teor de amorfismo (\%) & 88,0 & 87,7 & 83,7 & 68,2 \\
\hline
\end{tabular}

Mesmo a CCA950, que qualitativamente seria classificada como cristalina, apresenta em sua composição quase $70 \%$ de materiais reativos. Vale ressaltar que, para quantificação, a análise detalhada das fases presentes na amostra é fundamental. Além disso, deve-se buscar trabalhar sempre com banco de dados atuais que mostram-se mais completos. Este fato explica o aparecimento de fases que anteriormente não foram citadas.

No que diz respeito à quantificação de material amorfo da CCA1100, não foi possível chegar ao um ajuste que permitisse a quantificação das fases amorfas. Supõe-se que a mesma apresente uma grande quantidade de sílica na sua forma cristalina, fator este que impossibilitou um ajuste aceitável de um bom refinamento. A figura 10 apresenta a correlação entre o índice de amorfismo e as diferentes temperaturas de queimas.

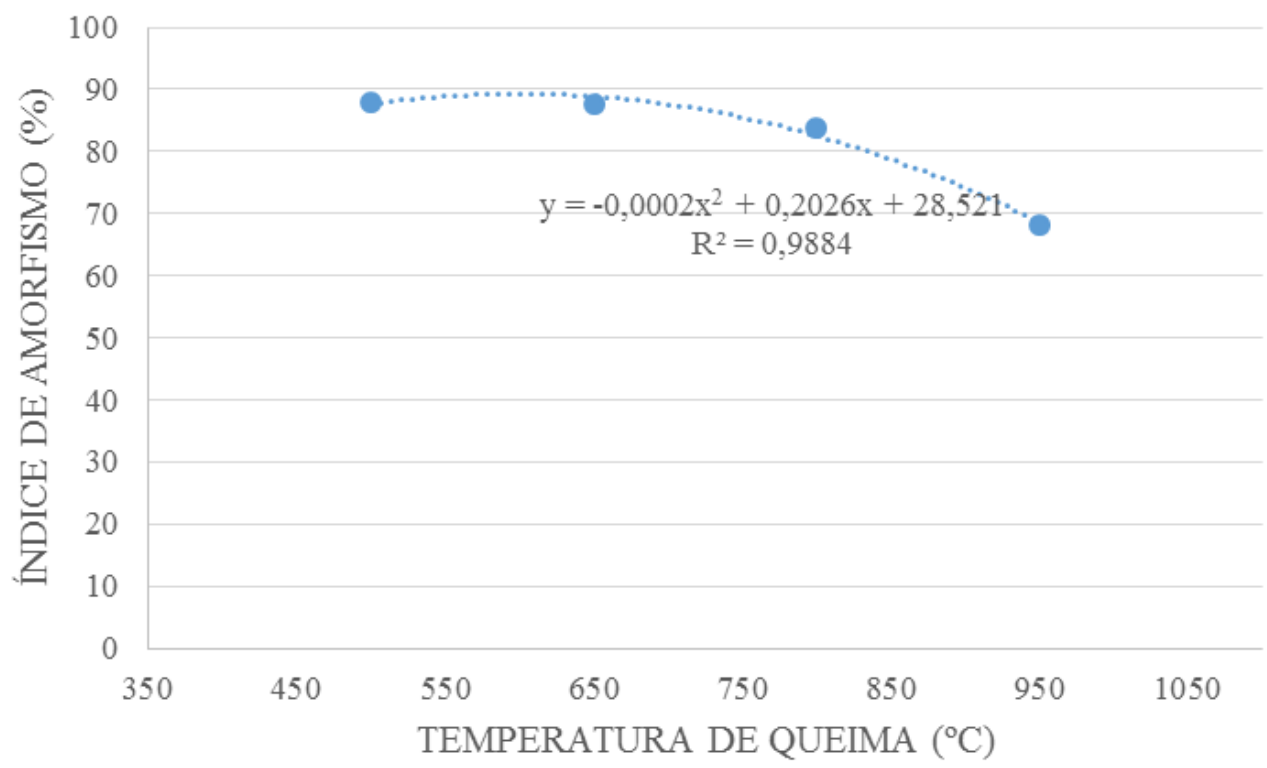

Figura 10: Correlação entre a temperatura de queima e o índice de amorfismo

As CCA por sua natureza apresentam uma grande quantidade de sílica em sua estrutura. Observa-se que a quantidade de sílica reativa diminui à medida que se aumenta a temperatura de queima; mesmo assim, a CCA apresenta sílica amorfa remanescente, em altas temperaturas, suficiente para garantir a reação pozolânica. Esse fator pode ser claramente observado na CCA950 que possui um teor amorfo de quase $70 \%$.

Feng et al [11]. (2004), também estudaram a porcentagem de material amorfo em diferentes CCA, só que por método químico. Os autores obtiveram para as cinzas produzidas em diferentes temperaturas $(550$, 600,700 e $800^{\circ} \mathrm{C}$ ) os valores de índice de amorfismo de 88,6\%,89,6\%, 75,4\% e 40,2\% respectivamente. 


\section{CONCLUSÕES}

A partir dos resultados obtidos neste trabalho conclui-se que o método de refinamento de Rietveld para a quantificação de fases reativas de CCA, apresentou resultados satisfatórios. E a técnica de refinamento de Rietveld pode ser usada como parâmetro de avaliação do potencial pozolânico de cinzas residuais.

A determinação do índice de amorfismo, através dessa técnica, mostrou-se prática e rápida. No entanto, é necessário um conhecimento mínimo de mineralogia para a realização da quantificação dos minerais e a quantificação do potencial reativo das amostras.

Percebeu-se também que só a análise semi-qualitatitiva não é suficiente para avaliar o potencial reativo de um resíduo com características pozolânicas. Como por exemplo, a CCA950 que qualitativamente apresenta uma grande quantidade de picos cristalinos, seria descartada em um primeiro momento por não ter sílica amorfa suficiente para promover as reações pozolânicas. No entanto, a CCA950 produzida nesta pesquisa apresentou quase $70 \%$ de sílica na sua forma amorfa, e produziu argamassas (CORDEIRO et al., 2009) com pozolanicidade suficiente para atender a NBR12653 (1992) e a ASTM C618 (2012).

\section{AGRADECIMENTOS}

A CAPES pelo apoio recebido para o desenvolvimento deste trabalho.

\section{BIBLIOGRAFIA}

[1] ASSOCIAÇÃO BRASILEIRA DE NORMAS TÉCNICAS. Materiais pozolânicos — Requisitos: NBR 12653. Rio de Janeiro, 2012.

[2] ASSOCIAÇÃO BRASILEIRA DE NORMAS TÉCNICAS. Materiais pozolânicos - Determinação da atividade pozolânica - Índice de atividade pozolânica com cal - Método de ensaio: NBR 5751. Rio de Janeiro, 2012.

[3] ASSOCIAÇÃO BRASILEIRA DE NORMAS TÉCNICAS. Materiais pozolânicos - Determinação de atividade pozolânica com cimento Portland - Índice 6de atividade pozolânica com cimento - Método de ensaio: NBR 5752. Rio de Janeiro, 2012.

[4] ASSOCIAÇÃO BRASILEIRA DE NORMAS TÉCNICAS. Resíduos sólidos - Classificação: NBR 10004. Rio de Janeiro, 2004.

[5] AMERICAN STANDARDS FOR TESTING MATERIALS, ASTM C-150- Standard Specification for Portland Cements, Philadelphia, EUA, 2012.

[6] ASTM. AMERICAN SOCIETY FOR TESTING AND MATERIALS. ASTM C618-05: Standard Specification for Coal Fly Ash and Raw or Calcined Natural Pozzolan for Use in Concrete. Philadelphia, EUA, 2012.

[7] BARTHA, P., HUPPERTZ, E.A. Structure and crystallization of silica in rice husk, In: Proceedings of the Rice By products Utilization International Conference, Valencia, Spain, 1974.

[8] CORDEIRO, G. C. Utilização de cinzas ultrafinas do bagaço de cana de açúcar e da casca de arroz como aditivos minerais em concreto. Tese de D.Sc.- Universidade Federal do Rio de Janeiro, Rio de Janeiro, 2006.

[9] CORDEIRO, L. N. P., MASUERO, A. B., DAL MOLIN, D.C.C. Análise da variação do índice de amorfismo sobre a atividade pozolânica. In: Congresso Brasileiro do Concreto, 53, Curitiba, 2009.

[10] DASS, A. Pozzolanicity of rice husk ash. In: The 9 CIB Congress, Stocholm - CIB-83. To builod and take care of what we have built with limited resources. Building Materials and Components, v. 4, p. 85-96, 1984.

[11] FENG. H.Q., YAMAMICHI. M., SUGITA, S. Study on the pozzolanic properties of rice husk ash by hydrochloric acid pretreatment, Cement and Concrete Research, v.34, p.521-526, 2004.

[12] HARA, N. et al. Hydrotermal Reactivity of Rice Husk Ash and its Use for Calcium Silicate Products. In: Fly Ash, Silica Fume, Slag and Natural Pozzolans in concrete, Trondheim, Normay. Proceedings...CAMMET, ACI SP-114, p. 499-516, 1989.

[13] NEVILLE, Adam M. Propriedades do Concreto. 2ed., São Paulo: Pini,1994.

[14] NEUMANN, R., SCHENEIDER, C. L., NETO, A. A., Caracterização Tecnológica de Minérios. Centro de Tecnologia Mineral. Comunicação Técnica elaborada para a $4^{\text {a }}$ Edição do Livro de Tratramento de Minérios. Rio de Janeiro, 2004. 
[15] PAULUK, S. Estudo da estrutura cristalina de pigmentos de zircônitas a partir dos sistemas ZrSiO4Co3O4 e $\mathrm{ZrSiO} 4-\mathrm{Cr} 2 \mathrm{O} 3$ utilizando o método de Rietveld. Dissertação de M.Sc. Química Aplicada, Ponta Grossa, 2008.

[16] SALVADOR, V.L.R. Separação de efeitos de sobreposição de espectros obtidos por WDXRF usando o método de Rietveld. Tese de D.Sc. em Tecnologia Nuclear-Materiais, São Paulo, 2005

[17] SENSALE, G. R. B. "Strength development of concrete with rice-husk ash", Cement and Concrete Composites, v.28, n.2, p.158-160, USA. 